Bull. Austral. Math. Soc.

$53 \mathrm{D} 12,35 \mathrm{~J} 60,35 \mathrm{~J} 65,53 \mathrm{C} 42,58 \mathrm{E} 12$

VOL. 76 (2007) [215-218]

\title{
A REMARK ON MINIMAL LAGRANGIAN DIFFEOMORPHISMS AND THE MONGE-AMPÈRE EQUATION
}

\section{JOHN URBAS}

We construct a counterexample to a theorem of Jon Wolfson concerning the existence of globally smooth solutions of the second boundary value problem for Monge-Amperre equations in two dimensions, or equivalently, on the existence of minimal Lagrangian diffeomorphisms between simply connected domains in $\mathbf{R}^{2}$.

In [6] Wolfson studied minimal Lagrangian diffeomorphisms between simply connected domains in $\mathbf{R}^{2}$. He derived several conditions guaranteeing the existence and nonexistence of such maps.

Given two bounded, connected, simply connected domains $D_{1}$ and $D_{2}$ in $\mathbf{R}^{2}$ with smooth boundaries $\partial D_{1}$ and $\partial D_{2}$, Wolfson calls the pair $\left(D_{1}, D_{2}\right)$ pseudoconvex if

$$
\min _{\partial D_{1}} \kappa_{1}+\min _{\partial D_{2}} \kappa_{2}>0
$$

where $\kappa_{1}, \kappa_{2}$ denote the curvatures of $\partial D_{1}, \partial D_{2}$ respectively relative to the inner normals.

One of his results states that if $\left(D_{1}, D_{2}\right)$ is a pseudoconvex pair of domains with equal areas, then there is a minimal Lagrangian diffeomorphism $\phi: \bar{D}_{1} \rightarrow \bar{D}_{2}$, smooth up to the boundary ([6, Theorem 5.1]).

An equivalent statement is that there is a solution $w \in C^{\infty}\left(\bar{D}_{1}\right)$ of the the second boundary problem for the Monge-Ampère equation

$$
\operatorname{det} \nabla^{2} w=1 \text { in } D_{1} \text {, }
$$

$$
\nabla w \text { is a diffeomorphism from } \bar{D}_{1} \text { onto } \bar{D}_{2} \text {, }
$$

([6, Corollary 6.2]).

These two statements are equivalent in the sense that if $\phi$ is a minimal Lagrangian diffeomorphism from $\bar{D}_{1}$ onto $\bar{D}_{2}$, then after a suitable choice of Lagrangian angle, $\phi$ can be written as $\nabla w$ where $w$ solves (2), and vice versa.

The existence of globally smooth solutions of (2) was proved by Delanoë [4] under the assumption that both $\partial D_{1}$ and $\partial D_{2}$ have positive curvatures. The higher dimensional analogue of Delanoë's result was proved by Caffarelli [3] and the author [5].

Received 18th December, 2006

Copyright Clearance Centre, Inc. Serial-fee code: 0004-9727/07 \$A2.00+0.00. 
Here we construct a counterexample to Wolfson's existence result.

THEOREM. There is a smooth, pseudoconvex pair of domains $\left(D_{1}, D_{2}\right)$ in $\mathbf{R}^{2}$ with equal areas such that there is no solution $w \in C^{2}\left(\bar{D}_{1}\right)$ of (2). Consequently, there is no globally smooth minimal Lagrangian diffeomorphism from $\bar{D}_{1}$ onto $\bar{D}_{2}$.

REMARK. It will be clear from the construction that $\frac{\min }{\partial D_{1}} \kappa_{1}+\min _{\partial D_{2}} \kappa_{2}$ can be made arbitrarily large.

The key to this construction is the following "obliqueness condition", the proof of which we defer to the end of the paper.

Lemma. Let $\nu_{1}$ and $\nu_{2}$ denote the inner unit normal vector fields to $\partial D_{1}$ and $\partial D_{2}$ respectively. Let $w \in C^{2}\left(\bar{D}_{1}\right)$ be a solution of (2). Then

$$
\nu_{1}(x) \cdot \nu_{2}(\nabla w(x))>0 \text { for all } x \in \partial D_{1}
$$

PROOF OF TheOREM: Consider the spiral $\gamma$ in $\mathbf{R}^{2}$ given in polar coordinates $(r, \theta)$ on $\mathbf{R}^{2}$ by $r(t)=1+t, \theta(t)=t$ for $t \in[0, \infty)$. This is a convex curve that starts at $(1,0)$ and spirals in the anticlockwise direction around the origin. The curvature of $\gamma$ relative to the inward pointing normal vector field $\nu$ (that is, towards the origin) is bounded between 0 and $3 / \sqrt{8}<2$. For $L>0$ to be fixed later let

$$
\Gamma_{L}=\operatorname{Image}\left(\left.\gamma\right|_{[0, L]}\right) \text {. }
$$

For small $\varepsilon>0$, also to be fixed later, let

$$
D_{\varepsilon, L}=\left\{x \in \mathbf{R}^{2}: \operatorname{dist}\left(x, \Gamma_{L}\right)<\varepsilon\right\}
$$

be the $\varepsilon$-neighbourhood of $\Gamma_{L}$. The area of $D_{\varepsilon, L}$ is approximately $2 \varepsilon \widetilde{L}+\pi \varepsilon^{2}$, where

$$
\widetilde{L}=\int_{0}^{L} \sqrt{1+(1+t)^{2}} d t
$$

is the length of $\Gamma_{L}$. Furthermore, $\partial D_{\varepsilon, L} \in C^{1,1}$ and the curvature of $\partial D_{\varepsilon, L}$ with respect to the inner normal vector field is bounded from below by $-2+O(\varepsilon)$. By smoothing $\partial D_{\varepsilon, L}$ near the two semicircular parts of its boundary we obtain a connected, simply connected domain $\widetilde{D}_{\varepsilon, L}$ with $\partial \widetilde{D}_{\varepsilon, L} \in C^{\infty}$ and such that

$$
\operatorname{area}\left(\widetilde{D}_{\varepsilon, L}\right)=2 \varepsilon \widetilde{L}, \quad \text { curvature of } \partial \widetilde{D}_{\varepsilon, L} \geqslant-3 .
$$

We now fix $L \geqslant 4 \pi$, so that $\Gamma_{L}$ winds around the origin at least twice and the Gauss map of $\Gamma_{L}$ covers every point in $\mathbf{S}^{1}$ at least twice. We then let $B=B_{r}(0)$ with $r>0$ chosen so that

$$
\operatorname{area}(B)=\operatorname{area}\left(\widetilde{D}_{\varepsilon, L}\right)
$$


Obviously $r \rightarrow 0$ as $\varepsilon \rightarrow 0$ for fixed $L$, so by making $\varepsilon$ small enough we can make the curvature of $\partial B$ as large as we want, say greater than 4 . Then $\left(D_{1}, D_{2}\right):=\left(\widetilde{D}_{\varepsilon, L}, B\right)$ is a pseudoconvex pair.

We now claim that there is no $C^{1}$ diffeomorphism $\psi$ from $\bar{D}_{1}$ onto $\bar{D}_{2}$ such that

$$
\nu_{1}(x) \cdot \nu_{2}(\psi(x))>0 \text { for all } x \in \partial D_{1} .
$$

Suppose on the contrary that there is such a diffeomorphism, and let $\xi=(-r, 0)$, $\eta=(r, 0)$ where $r>0$ is as above. Let $\gamma^{+}$and $\gamma^{-}$denote the closed upper and lower semicircles of $\partial D_{2}$. Let $\hat{\xi}=\psi^{-1}(\xi), \widehat{\eta}=\psi^{-1}(\eta)$ and $\hat{\gamma}^{+}=\psi^{-1}\left(\gamma^{+}\right), \hat{\gamma}^{-}=\psi^{-1}\left(\gamma^{-}\right)$. From the construction of $D_{1}$ and the fact that $\left.\psi\right|_{\partial D_{1}}$ is a diffeomorphism from $\partial D_{1}$ onto $\partial D_{2}$, we see that the Gauss map of at least one of the curves $\hat{\gamma}^{+}$and $\widehat{\gamma}^{-}$must cover $\mathbf{S}^{1}$. If this curve is $\widehat{\gamma}^{+}$, then (4) implies that $(0,-1)$ does not belong to $\nu_{1}\left(\widehat{\gamma}^{+}\right)$, while if the curve is $\hat{\gamma}^{-}$, then (4) implies that $(0,1)$ does not belong to $\nu_{1}\left(\hat{\gamma}^{-}\right)$. In either case we obtain a contradiction.

Proof Of Lemma: This result is proved in [5]. Since the proof is short we include it here for the convenience of the reader.

Let $w \in C^{2}\left(\bar{D}_{1}\right)$ be a solution of (2). Since $D_{1}$ is connected, either $\nabla^{2} w>0$ everywhere or $\nabla^{2} w<0$ everywhere; in either case $\nabla^{2} w$ is invertible. Let $h \in C^{1}\left(\bar{D}_{2}\right)$ be a function such that $h>0$ in $D_{2}$ and $h=0,|\nabla h|=1$ on $\partial D_{2}$. Then $H=h(\nabla w)$ is positive in $D_{1}$ and zero on $\partial D_{1}$, so

$$
\nabla_{\tau} H=h_{p_{k}} \nabla_{k \tau} w=0 \quad \text { on } \quad \partial D_{1}
$$

for any tangential vector field $\tau$ on $\partial D_{1}$, and

$$
\nabla_{\nu} H=h_{p_{k}} \nabla_{k \nu} w \geqslant 0 \text { on } \partial D_{1},
$$

where to simplify notation we write $\nu$ rather than $\nu_{1}$ for the inner unit normal vector field to $\partial D_{1}$. Thus

$$
\nabla_{i} H=h_{p_{\boldsymbol{h}}} \nabla_{i k} w=\left(\nabla_{\nu} H\right) \nu_{i} \text { on } \partial D_{1} .
$$

Since $\nabla^{2} w$ is invertible, we see that

$$
\chi:=h_{p_{k}} \nu_{k}=\left(\nabla_{\nu} H\right) w^{\nu \nu} \text { on } \partial D_{1},
$$

where $w^{\nu \nu}=w^{i j} \nu_{i} \nu_{j}$ and $\left[w^{i j}\right]=\left[\nabla^{2} w\right]^{-1}$. From (5) we also see that

$$
h_{p_{i}} h_{p_{k}} \nabla_{i k} w=\chi \nabla_{\nu} H \text {. }
$$

Combining this with (6) we obtain

$$
\chi=\sqrt{w^{i j} \nu_{i} \nu_{j} \nabla_{k l} w h_{p_{k}} h_{p_{l}}} \text { on } \partial D_{1}
$$


which is positive since $w \in C^{2}\left(\bar{D}_{1}\right)$ with either $\nabla^{2} w>0$ or $\nabla^{2} w<0$ in $\bar{D}_{1}$. Finally, we observe that $\nabla h_{\partial D_{2}}=\nu_{2}$, so $\chi(x)=\nu_{1}(x) \cdot \nu_{2}(\nabla w(x))$ and (3) follows.

REMARK. Brenier [1] has shown that given any two bounded domains $D_{1}, D_{2} \subset \mathbf{R}^{n}$ with $\left|D_{1}\right|=\left|D_{2}\right|$ and $\left|\partial D_{1}\right|=\left|\partial D_{2}\right|=0$ (where $|\cdot|$ denotes Lebesgue measure in $\mathbf{R}^{n}$ ), there is a convex function $u$ (unique up to constants) such that

$$
\operatorname{det} \nabla^{2} u=1 \quad \text { in } \quad D_{1}, \quad \nabla u\left(D_{1}\right)=D_{2}
$$

in a suitable generalised sense, where the equation is interpreted in an integral sense and $\nabla u$ is interpreted in the almost everywhere sense. Moreover, Caffarelli [2] has proved the interior regularity of convex Brenier solutions of (7) if $D_{2}$ is convex. Thus our example shows that it is the global regularity, not the existence or interior regularity, that may fail under Wolfson's pseudoconvexity condition.

\section{REFERENCES}

[1] Y. Brenier, 'Polar factorization and monotone rearrangement of vector valued functions', Comm. Pure Appl. Math. 44 (1991), 375-417.

[2] L. Caffarelli, 'The regularity of mappings with a convex potential', J. Amer. Math. Soc. 5 (1992), 99-104.

[3] L. Caffarelli, 'Boundary regularity of maps with convex potentials II', Ann. of Math. 144 (1996), 453-496.

[4] P. Delanoë, 'Classical solvability in dimension two of the second boundary value problem associated with the Monge-Ampère operator', Ann. Inst. H. Poincaré Anal. Non Linéaire 8 (1991), 443-457.

[5] J. Urbas, 'On the second boundary value problem for equations of Monge-Ampère type', J. Reine Angew. Math. 487 (1997), 115-124.

[6] J. Wolfson, 'Minimal Lagrangian diffeomorphisms and the Monge-Ampère equation', $J$. Differential Geom. 46 (1997), 335-373.

Centre for Mathematics and its Applications

Mathematical Sciences Institute

Australian National University

Canberra ACT 0200

Australia

e-mail: urbas@maths.anu.edu.au 